\title{
Combinations of Service Use Types of People With Early Cognitive Disorders
}

Citation for published version (APA):

Janssen, N., Handels, R. L. H., Koehler, S., Ramakers, I. H. G. B., Hamel - de Bakker, R., Rikkert, M. G. M. O., Scheltens, P., Bouwman, F. H., van der Flier, W. M., Wolfs, C. A. G., de Vugt, M. E., Evers, S. M. A. A., \& Verhey, F. R. J. (2016). Combinations of Service Use Types of People With Early Cognitive Disorders. Journal of the American Medical Directors Association, 17(7), 620-625.

https://doi.org/10.1016/j.jamda.2016.02.034

Document status and date:

Published: 01/07/2016

DOI:

10.1016/j.jamda.2016.02.034

Document Version:

Publisher's PDF, also known as Version of record

Document license:

Taverne

Please check the document version of this publication:

- A submitted manuscript is the version of the article upon submission and before peer-review. There can be important differences between the submitted version and the official published version of record.

People interested in the research are advised to contact the author for the final version of the publication, or visit the DOI to the publisher's website.

- The final author version and the galley proof are versions of the publication after peer review.

- The final published version features the final layout of the paper including the volume, issue and page numbers.

Link to publication

\footnotetext{
General rights rights.

- You may freely distribute the URL identifying the publication in the public portal. please follow below link for the End User Agreement:

www.umlib.nl/taverne-license

Take down policy

If you believe that this document breaches copyright please contact us at:

repository@maastrichtuniversity.nl

providing details and we will investigate your claim.
}

Copyright and moral rights for the publications made accessible in the public portal are retained by the authors and/or other copyright owners and it is a condition of accessing publications that users recognise and abide by the legal requirements associated with these

- Users may download and print one copy of any publication from the public portal for the purpose of private study or research.

- You may not further distribute the material or use it for any profit-making activity or commercial gain

If the publication is distributed under the terms of Article $25 \mathrm{fa}$ of the Dutch Copyright Act, indicated by the "Taverne" license above, 
Original Study

\title{
Combinations of Service Use Types of People With Early Cognitive Disorders
}

\author{
Niels Janssen MSc ${ }^{\mathrm{a}}$, Ron L.H. Handels PhD ${ }^{\mathrm{a}}$, Sebastian Koehler PhD ${ }^{\mathrm{a}}$, \\ Inez H.G.B. Ramakers PhD ${ }^{\mathrm{a}}$, Renske E.G. Hamel PhD ${ }^{\mathrm{a}}$, \\ Marcel G.M. Olde Rikkert MD, PhD ${ }^{\mathrm{b}}$, Philip Scheltens MD, PhD ${ }^{\mathrm{c}}$, \\ Femke H. Bouwman MD, PhD ${ }^{c}$, Wiesje M. van der Flier PhD ${ }^{\mathrm{c}, \mathrm{d}}$, Claire A.G. Wolfs PhD ${ }^{\mathrm{a}}$, \\ Marjolein E. de Vugt PhD ${ }^{\mathrm{a}}$, Silvia M.A.A. Evers PhD ${ }^{\mathrm{e}}$, Frans R.J. Verhey MD, PhD ${ }^{\mathrm{a}, *}$ \\ a Alzheimer Center Limburg, School for Mental Health and Neuroscience, Maastricht University Medical Center, Maastricht, The Netherlands \\ ${ }^{\mathrm{b}}$ Department of Geriatrics and Radboud Alzheimer Center, Radboud University Medical Center, Nijmegen, The Netherlands \\ ${ }^{c}$ Alzheimer Center, Department of Neurology, VU University Medical Center, Amsterdam, The Netherlands \\ ${ }^{\mathrm{d}}$ Department of Epidemiology and Biostatistics, VU University Medical Center, Amsterdam, The Netherlands \\ ${ }^{\mathrm{e}}$ Department of Health Services Research, School for Public Health and Primary Care, Maastricht University, The Netherlands
}

\section{Keywords:}

Early diagnosis

service use

dementia

mild cognitive impairment

latent class analysis

\section{A B S T R A C T}

Objectives: Understanding which persons most likely use particular combinations of service types is important as this could lead to a better understanding of care pathways. The aim of this study is to identify combinations of service use within a sample of community-dwelling people with mild cognitive impairment (MCI) and dementia and identify factors related to these service use combinations. Methods: A latent class analysis performed at baseline on a merged dataset $(\mathrm{n}=530)$ was used to classify care recipients based on following service use types: general practitioner visits, physiotherapist visits, hospital outpatient specialist visits, emergency room visits, hospital inpatient visits with stay over, day care visits, use of domestic homecare, use of personal homecare, and informal care on (instrumental) activities of daily living. Multinomial logistic regression was performed to identify factors associated with service use combinations using clinical characteristics of the care recipient and demographic characteristics of the care recipient and caregiver.

Results: Three service use classes were identified; a formal homecare class (10\% of participants), an informal care class ( $46 \%$ of participants), and a low user class (44\% of participants). Factors increasing the likelihood of being in the formal homecare class compared with the low service use class included a diagnosis of MCI or dementia, activities of daily living impairment, older age of the care recipient, and care recipient not living together with the caregiver.

Conclusions: Besides a diagnosis of MCI or dementia, other factors (activities of daily living impairment, age, and living situation) were associated with service use. We recommend using these factors alongside the diagnostic label for care indication.

(c) 2016 AMDA - The Society for Post-Acute and Long-Term Care Medicine.
Alzheimer disease (AD) and associated dementia disorders have a great impact on people with dementia and their families. With a worldwide prevalence of 36 million $^{1}$ and related cost of $\$ 604$ billion, ${ }^{2}$ dementia places a substantial burden on societies. Because of the progressive nature of the disease, people with dementia often require

\footnotetext{
The authors declare no conflicts of interest.

* Address correspondence to Frans R.J. Verhey, MD, PhD, Alzheimer Center Limburg, School for Mental Health and Neuroscience, Maastricht University Medical Center, P.O. Box 5800, Maastricht AZ 6202, The Netherlands.

E-mail address: f.verhey@maastrichtuniversity.nl (F.R.J. Verhey).
}

increasing amounts of support on their cognitive abilities and activities of daily living (ADL) at home and often eventually in an institution. $^{3,4}$ Early diagnosis of dementia is considered important for access to treatment, support, and future care and life planning for persons with dementia and their caregivers. ${ }^{1,5}$

It is unclear what types of services are being used after a formal early diagnosis has been made. Understanding how clinical and demographic characteristics influence the use of care services is important to plan timely access to these types of care. Furthermore, because many different care providers are involved in the diagnostic process and care provision, insight in factors related to service use 
types could lead to better coordination of care between providers and informal caregivers.

Several studies examined service use of people with dementia and their caregivers. Overall, a diagnosis of dementia has been found to be a major determinant of service use ${ }^{6}$ with increasing service use in more severe disease stages. ${ }^{6-8}$ People with dementia use healthcare services (eg, specialist visits) more often than community services (eg, day care). ${ }^{9-11}$ Factors associated with higher levels of service use have been found to be impaired $\mathrm{ADL},{ }^{6,7,12}$ neuropsychiatric symptoms, ${ }^{4}$ comorbidities, ${ }^{8}$ not having a spousal caregiver, ${ }^{8,13}$ caregiver and person with dementia not living together, ${ }^{13,14}$ knowledge of available services, ${ }^{15-17}$ caregiver's positive attitude toward service use, ${ }^{18}$ higher caregiver burden, ${ }^{17}$ higher number of skilled nursing facilities, ${ }^{19}$ availability of public and private transportation, ${ }^{13}$ higher educational level, ${ }^{8,13}$ higher age, and not being married. ${ }^{6}$ Factors for non-utilization of services have been found to be severity of cognition and a negative attitude of the person with dementia toward service use. ${ }^{17}$ Furthermore, Beeber et $\mathrm{al}^{19}$ showed that people with dementia and their caregivers tend to use a combination of different types (eg, using home health and home aide) rather than a single service type.

Studies on service use of people with mild cognitive impairment $(\mathrm{MCI})$, often preceding dementia ${ }^{20}$ are scarce. Although $\mathrm{MCI}$ according to the criteria is not severe enough to interfere with activities of daily living, ${ }^{21}$ people with $\mathrm{MCI}$ showed to have higher medical costs and receive more informal care compared to people without $\mathrm{MCI}^{22}$ This finding suggests that informal care already starts before a person develops dementia.

Current evidence mainly focuses on associations with single types of service use. Only Beeber et $\mathrm{al}^{19}$ focused on combinations of service types, although limited to community services. More research is needed to identify combinations of service types when incorporating a broader range of service types.

A better understanding of combinations and associated factors is important for timely access and coordination of care for people with MCI and dementia. This cross-sectional study, therefore, aims to identify subgroups of community-dwelling patients with $\mathrm{MCI}$ and dementia who share similar combinations of service use at their initial visit to a memory clinic using latent class analysis (LCA), and examine which clinical and demographic factors are related to these subgroups.

\section{Methods}

\section{Design}

A cross-sectional secondary data analysis was carried out on a merged dataset with data of 4 Dutch longitudinal studies with overlapping protocols: (1) the Leiden Alzheimer Research Netherlands (LEARN) study, ${ }^{23}$ (2) the Clinical Course of Cognition and Comorbidity (4C) MCI study (Liao W, Hamel RE, Olde Rikkert MG, et al. Cohort profile: The Clinical Course of Cognition and Comorbidity in Mild Cognitive Impairment and Dementia [the 4C study]: Two complementary longitudinal, clinical cohorts in The Netherlands, unpublished data), (3) the 4C Dementia study ${ }^{24}$ (data from Maastricht location only), and (4) the Dutch Flutemetamol study. ${ }^{25}$ In all studies, patients were referred to the memory clinic of 1 of 4 academic hospitals (Maastricht, Leiden, Nijmegen, or Amsterdam) for evaluation of their cognitive complaints. They received an extensive clinical examination and the informal caregiver was asked to fill out a booklet with questions about service use, informal care, working situation and quality of life of themselves and the person with the memory disorder.

\section{Study Population}

Inclusion criteria of the Clinical Course of Cognition and Comorbidity in Mild Cognitive Impairment (4C-MCI) and Clinical Course of
Cognition and Comorbidity in Dementia (4C-Dementia), LEARN, and the Dutch Flutemetamol study consisted of (1) a Clinical Dementia Rating score of 0-2; (2) a Mini-Mental State Examination (MMSE) score of $\geq 10$; and ( 3 ) the availability of having a reliable proxy, further referred to as the informal caregiver.

Exclusion criteria were other neurologic diseases (ie, normal pressure hydrocephalus, Parkinson disease, Huntington disease, cognitive problems because of alcohol use, a cerebral vascular accident or transient ischemic attack less than 2 years ago, brain tumor, epilepsy, encephalitis); a psychiatric history less than 12 months ago (ie, major depression according to the Statistical Manual of Mental Disorders, 4th Edition); and suspicion of the participants not being able to have at least 1 follow-up.

In total, 1033 people participated in 4 studies: 304 in the LEARN study, 315 in the 4C MCI study, 329 in the 4C Dementia study, and 211 in the Dutch Flutemetamol study (some people participated in more than 1 study, $\mathrm{n}=126$ ). Of all 1033 participants, 178 participated solely in 4C Dementia study in Amsterdam or Nijmegen of whom service use data was not obtained. Participants were excluded because of a diagnosis of subjective memory complaints $(n=199)$, being institutionalized ( $\mathrm{n}=55$ ), having all clinical data or the syndrome diagnosis missing $(n=2)$, or having all service use data missing $(n=104)$. The final sample eligible for analyses comprised 530 community-dwelling people with $\mathrm{MCI}$ or dementia, further referred to as care recipients.

\section{Measures}

Demographic characteristics of the care recipients included gender, age, and years of formal education. Demographic characteristics of the informal caregivers included gender, age, years of formal education, living situation, and working situation. Clinical measures of the care recipients included a diagnosis of $\mathrm{MCI}$ or dementia (because of $\mathrm{AD}$ or other cause), cognitive functioning measured by the MMSE, with lower scores representing more severe cognitive problems, ${ }^{26}$ behavioral problems measured by the neuropsychiatric inventory (NPI), with higher scores indicating increased severity ${ }^{27}$; and functional abilities of ADL measured by the disability assessment for dementia (DAD), with lower scores indicating increased disabilities on performing ADL. ${ }^{28}$

Measures of service use were derived through a comprised questionnaire filled out by the informal caregiver. ${ }^{23}$ This questionnaire consisted of the Resource Utilization in Dementia Lite ${ }^{29}$ and additional questions, which measured service use on general practitioner (GP) visits, physiotherapy visits, psychologist visits, community mental health team visits, emergency room visits, hours of personal or nursing homecare, hours of domestic homecare, days of day care at nursing home, care home or community center, being admitted to a nursing home or care home, hospital outpatient specialist visits (any-type eg, geriatrician, urologist), hospital inpatient visits with stay overnight, hospital inpatient visits without stay overnight, hours of informal care of activities in daily living (ADL; eg, dressing), and hours of informal care of instrumental ADL (IADL; eg, cooking). In the questionnaire it was asked if and how often the care recipient, or the informal caregiver because of the problems of the patient, used these services within a recall period of 3 months. A copy of the booklet can be provided upon request.

The following service use variables were included for further analyses: GP visits, physiotherapy visits, emergency room visits, personal homecare, domestic homecare, day care visit, hospital outpatient specialist visits, hospital inpatient visits with overnight stay and informal care ADL, and informal care IADL. Psychologist visits, community mental health team visits, and hospital inpatient visits without stay-over were excluded because data were only obtained in a subsample. 
Table 1

Demographic and Clinical Characteristics of Patients With MCI and Dementia and Their Informal Caregivers at First Visit to Memory Clinic Using Nonimputed Data

\begin{tabular}{|c|c|c|c|c|}
\hline Demographics & $\begin{array}{l}\mathrm{MCI}(\mathrm{n}=198,37 \%) \\
\text { Mean }(\mathrm{SD}) \text { Range }\end{array}$ & $\begin{array}{l}\text { Dementia ( } \mathrm{n}=332,63 \%) \\
\text { Mean (SD) Range }\end{array}$ & Missing (\%) & Significance \\
\hline \multicolumn{5}{|l|}{ Patient } \\
\hline Age, years & $69.7(8.8) 50-89$ & 69.5 (10) 41-91 & 0 & .763 \\
\hline Gender, male (\%) & $61 \%$ & $53 \%$ & 0 & .080 \\
\hline Years of education & 10.9 (3.6) 6-17 & 11.0 (3.7) 6-17 & 0 & .729 \\
\hline MMSE & $26.3(2.6) 18-30$ & 23.0 (3.4) $13-30$ & 1 & .000 \\
\hline $\mathrm{DAD}$ & $86.6(13.8) 38-100$ & 75.2 (23.7) $0-100$ & 13 & .000 \\
\hline NPI, total score & $14.0(14.5) 0-76$ & $19.2(17.9) 0-88$ & 37 & .004 \\
\hline \multicolumn{5}{|l|}{ Informal caregiver } \\
\hline Age, years & 62 (11.7) 17-86 & $61.7(10.8) 22-90$ & 3 & .763 \\
\hline Gender, male (\%) & $35 \%$ & $36 \%$ & 1 & .894 \\
\hline Years of education & $11.4(3.1) 6-17$ & $11.7(3.2) 6-17$ & 4 & .279 \\
\hline Lives together with patient (\%) & $76 \%$ & $72 \%$ & 2 & .351 \\
\hline Having a paid job (\%) & $36 \%$ & $43 \%$ & 2 & .105 \\
\hline
\end{tabular}

${ }^{*}$ Independent $t$ test for continuous data and Pearson $\chi^{2}$ test for proportions.

\section{Statistical Analyses}

LCA was conducted to identify combinations of service use types. LCA is used to detect the smallest number of latent classes by grouping individual care recipients into categories based on similarities in service use types. This was done by starting with a 1-class model and stepwise increasing the number of classes. The estimation of the number of classes was based on information criteria, likelihood ratio tests using the three step approach, ${ }^{30}$ entropy score, and researcher's interpretation. The Bayesian Information Criterion ${ }^{31}$ considers the model with the lowest value to be the superior model. The LoMendell-Rubin test ${ }^{32}$ examined if the estimated model fitted significantly better than the model with 1 class less $^{33}$ using a $P$ value of .05 . Entropy scores, ranging from 0 to 1 , were used as an indicator of classification certainty using a cut-off score of $>0.80$. All class models were eventually reanalyzed using a parametric bootstrapped likelihood ratio test. ${ }^{34}$ This test is considered more robust than the LoMendell-Rubin test ${ }^{35}$ and should be significant at a $P$ value level of .05. Above all, interpretation of the meaningfulness of the latent class model was conclusive. The LCA was performed using Mplus v 7.3 (Muthén \& Muthén, Los Angeles, CA). ${ }^{36}$

Of the 530 participants, $63.4 \%$ had complete service use data, $33.4 \%$ had 1-3 missing variables, and $3.2 \%$ had more than 3 missing variables on service use. For the LCA, missing values on service use data were handled by the latent class analyses in Mplus 7.3 through the default option.

Conditional probabilities, or probabilities of using specific services given class membership, were plotted alongside their class proportion. Conditional probabilities were classified as low (0\%-40\%), moderate (41\%-69\%) and high, 70\%-100\%. ${ }^{37}$
Next, it was tested if class membership was associated with demographic and clinical characteristics. This was done by a multinomial logistic regression model on the saved individual most likely class membership in SPSS v 22 (IBM Corporation Armonk, NY). ${ }^{38}$ Because of the explorative nature of the study, first univariate analyses were performed to select independent variables. Significant variables $(P<.10)$ with less than $25 \%$ missing values were used in the multivariable analyses. Furthermore, colinearity between independent variables was assessed. The multinomial logistic regression was performed in a block wise fashion, starting with the syndrome and etiology diagnosis, then including the clinical characteristics MMSE, NPI, and DAD score, and the final block contained the informal caregivers' demographics age, gender, years of education, living situation and working situation. Model improvement was examined based on the significance $(P<.05)$ of the $\chi^{2}$ change.

Missing values on clinical and demographic characteristics were imputed through a multiple imputation procedure. In total $10 \mathrm{impu-}$ tations were performed.

\section{Results}

Baseline characteristics are displayed in Table 1. Most patients had a diagnosis of dementia (63\%), of which $65 \%$ had dementia because of $\mathrm{AD}$. The mean age of the care recipients was 70 years in the MCI group and 70 years in the dementia group. More than one-half of them were male (61\% for MCI and 53\% for dementia). The informal caregivers of the persons with $\mathrm{MCI}$ and dementia had a mean age of 62 years $(\mathrm{MCI})$ and 62 years (dementia), of whom less than one-half were male (35\% for MCI and 36\% for dementia). The $t$ tests and $\chi^{2}$ tests showed

Table 2

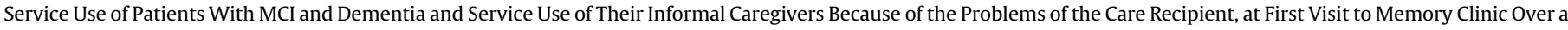
Recalled Period of 2 Weeks to 3 Months

\begin{tabular}{|c|c|c|c|c|c|c|}
\hline \multirow[t]{2}{*}{ Service Use Visits Past 3 Months } & \multicolumn{3}{|c|}{$\mathrm{MCI}(\mathrm{n}=198)$} & \multicolumn{3}{|c|}{ Dementia $(\mathrm{n}=332)$} \\
\hline & N (\%) Users & Median (Range) & Missing (\%) & $\mathrm{N}(\%)$ Users & Median (Range) & Missing (\%) \\
\hline GP & $153(77)$ & $2(1-20)$ & 1 & $243(73)$ & $2(1-30)$ & 3 \\
\hline Physiotherapist & $50(25)$ & $6(1-48)$ & 4 & $72(22)$ & $6(1-26)$ & 2 \\
\hline Hospital outpatient & $157(79)$ & $2(1-15)$ & 1 & $241(73)$ & $3(1-19)$ & 2 \\
\hline Inpatient stay over & $9(5)$ & $2(1-9)$ & 3 & $12(4)$ & $3(1-21)$ & 3 \\
\hline Emergency room & $15(8)$ & $1(1-4)$ & 4 & $25(8)$ & $1(1-3)$ & 5 \\
\hline Day care & $1(1)$ & $7(-)$ & 2 & $12(4)$ & $15.5(0.66-48)$ & 3 \\
\hline Domestic homecare* & $23(12)$ & $3.1(0.33-11.25)$ & 1 & $59(18)$ & $3.4(1-9.38)$ & 2 \\
\hline Personal homecare* & $18(9)$ & $6(0.37-14)$ & 2 & $27(8)$ & $5.75(1-19.38)$ & 2 \\
\hline Informal care ADL* & $43(22)$ & $7(0.03-112)$ & 7 & $122(37)$ & $7(0.03-112)$ & 2 \\
\hline Informal care IADL* & $78(39)$ & $7(0.10-112)$ & 8 & 209 (63) & $7(0.07-112)$ & 1 \\
\hline
\end{tabular}

*Hours per week instead of visits past 3 months. 
Table 3

Fit Statistics for Different Latent Class Models

\begin{tabular}{|c|c|c|c|c|c|c|}
\hline & 1 Class & 2 Class & 3 Class & 4 Class & 5 Class & 6 Class \\
\hline Log likelihood & -2189.865 & -2026.110 & -1988.720 & -1973.734 & -1961.322 & -1950.937 \\
\hline $\mathrm{BIC}$ & 4442.459 & 4183.950 & 4178.172 & 4217.202 & 4261.380 & 4309.610 \\
\hline Entropy & $\mathrm{n} / \mathrm{a}$ & 0.754 & 0.892 & 0.905 & 0.890 & 0.842 \\
\hline LMR & $\mathrm{n} / \mathrm{a}$ & .0000 & .0039 & .0257 & .5562 & .4642 \\
\hline BLR & $\mathrm{n} / \mathrm{a}$ & .0000 & .0000 & .0128 & .1333 & .4286 \\
\hline
\end{tabular}

BIC, Bayesian Information Criterion; BLR, bootstrap likelihood ratio test; LMR, Lo-Mendell-Rubin test; n/a, not applicable.

significant differences on the clinical variables MMSE, DAD, and NPI score between people with MCI and dementia (Table 1).

Table 2 summarizes the usage of services. Three cases showed extreme values of 168 hours per week for informal care ADL and IADL and were, therefore, transformed to 112 hours per week. During the recall period of 3 months, more than one-half of the participants visited their GP $(77 \% \mathrm{MCI}$, median visits $=2 ; 73 \%$ dementia, median visits $=2$ ) and visited outpatient specialist services (79\% MCI, median visits $=2 ; 73 \%$ dementia, median visits $=3$ ). Other services were used less frequently, except for the informal care IADL. More than one-half of the people with dementia used informal care IADL (63\%), with a median of 7 hours per week.

$L C A$

The results of the model selection criteria (Table 3 ) indicated that the 3-class model was superior to the other models. The 3-class model showed the lowest Bayesian Information Criterion value, a significant Lo-Mendell-Rubin test $(P<.05)$ and bootstrap likelihood ratio test $(P<.05)$, and had a high entropy score $(0.892)$. Furthermore, the 3 classes resulted in meaningful interpretation by showing qualitative and quantitative difference in type and frequency of service use among classes (Figure 1). GP visits and outpatient visits showed a high probability in all three classes. Probably most of these visits entailed a referral to the memory clinic. Class 1 contained $10 \%$ of the participants $(\mathrm{n}=54)$. Members of class 1 had a high probability of visiting the GP, outpatient visits, receiving informal care for IADL and using domestic homecare, a moderate probability of using personal homecare, and receiving informal care for $\mathrm{ADL}$, but a low probability of visiting physiotherapist, inpatient visits, emergency room visits, and day care visits. Class 1 was, therefore, labeled as the "formal homecare" class. Class 2 contained $46 \%$ of participants $(n=242)$. Members of class 2 had a high probability of visiting the GP, outpatient visits and receiving informal care for IADL, a moderate probability of receiving informal care for ADL, but a low probability for using the other service use types. Class 2 was labeled as the "informal care" class. Class 3 contained $44 \%$ of participants $(n=234)$ and showed a high probability of visiting the GP and outpatient specialists, and a low probability of using the other service use types. Despite the high probability of visiting the GP and outpatient specialists, class 3 was labeled as the "low-user" class.

\section{Multinomial Logistic Regression}

The method of using the most likely class as outcome in further analyses was appropriate to use because entropy score was high. ${ }^{39}$ Univariate analyses showed no significant results for the predictor variables age of the informal caregiver, gender of the informal caregiver, gender of the care recipient, informal caregiver's years of education, and working situation of the informal caregiver. NPI was missing in $37 \%$ of the cases and, therefore, excluded for this analyses. Complete case analysis $(\mathrm{n}=317)$ was performed including NPI. Results showed that NPI was not significant $(P=.154)$.

Table 4 shows the results of the multinomial logistic regression in terms of pooled odds ratios (ORs). Every block showed a significant improvement compared with the previous block.

Results of the final block showed that a higher age of the care recipient [OR 1.11; confidence interval $(\mathrm{CI})$ 1.06-1.16] increased the likelihood of being in the formal homecare class compared with the low user class, whereas a higher score on the DAD (OR 0.94; CI

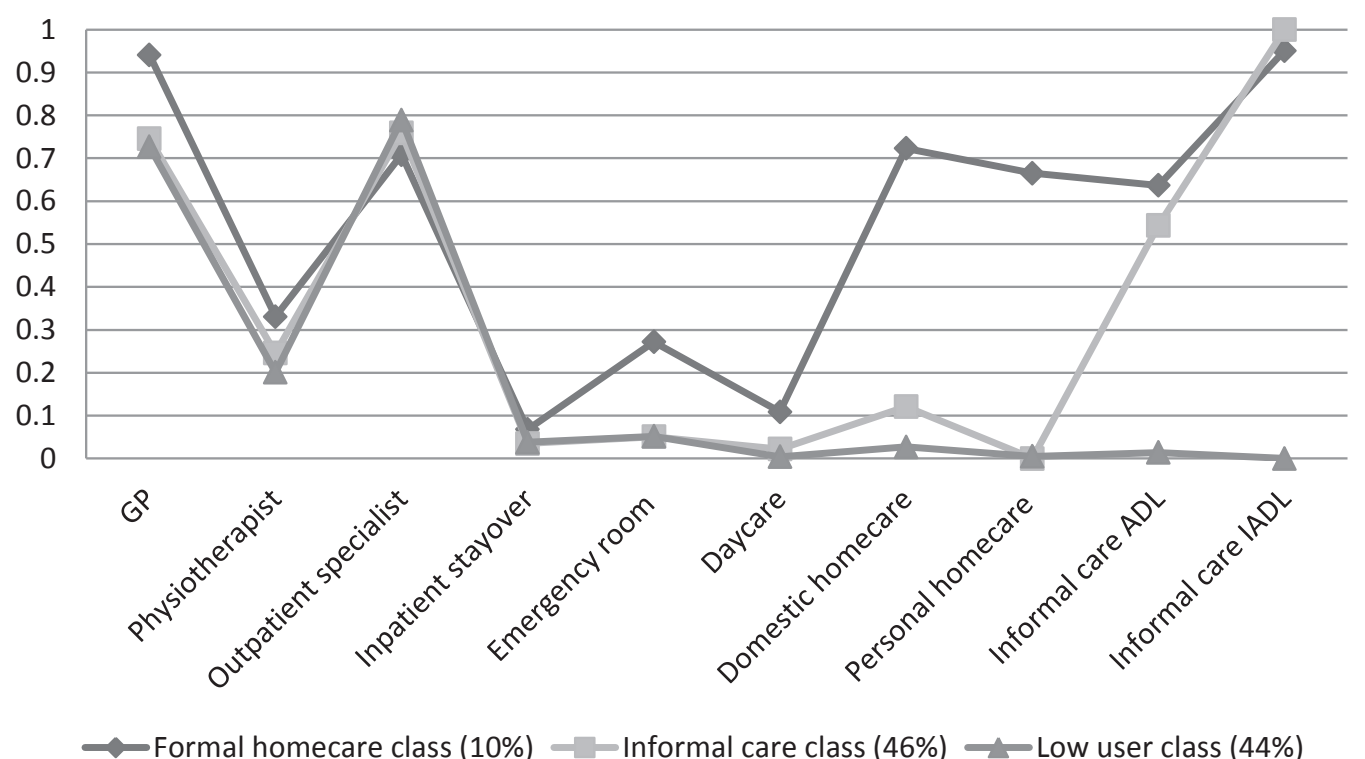

Fig. 1. Conditional probabilities (y-axis) and class proportions (x-axis). 
Pooled ORs From Multinomial Logistic Regression Predicting Class Membership

\begin{tabular}{|c|c|c|c|c|}
\hline & \multirow{2}{*}{$\frac{\text { Overall Group Differences }}{P \text { Value }}$} & \multicolumn{3}{|l|}{ Group Comparisons } \\
\hline & & $\begin{array}{l}\text { Formal Homecare } \\
\text { Class vs Low User Class }\end{array}$ & $\begin{array}{l}\text { Informal Care Class vs } \\
\text { Low User Class }\end{array}$ & $\begin{array}{l}\text { Formal Homecare vs } \\
\text { Informal Care Class }\end{array}$ \\
\hline Gender, male* & $>.05$ & $0.93(0.41-2.07)$ & $0.94(0.62-1.43)$ & $0.98(0.46-2.11)$ \\
\hline Age* $^{*}$ & $<.01$ & $1.11^{\dagger}(1.06-1.16)$ & $1.01(0.98-1.03)$ & $1.10^{\dagger}(1.05-1.15)$ \\
\hline Years of education* & $>.05$ & $0.91(0.81-1.02)$ & $0.95(0.90-1.01)$ & $0.96(0.85-1.07)$ \\
\hline Diagnosis & $<.01$ & & & \\
\hline AD vs $\mathrm{MCI}$ & & $0.48(0.18-1.29)$ & $1.84^{\ddagger}(1.13-2.99)$ & $0.26^{\dagger}(0.10-0.68)$ \\
\hline Other NDD vs MCI & & $1.31(0.47-3.61)$ & $2.16^{\dagger}(1.22-3.83)$ & $0.61(0.23-1.57)$ \\
\hline MMSE & $>.05$ & $0.96(0.85-1.09)$ & $0.97(0.91-1.04)$ & $0.99(0.86-1.11)$ \\
\hline $\mathrm{DAD}$ & $<.01$ & $0.94^{\dagger}(0.92-0.96)$ & $0.97^{\dagger}(0.95-0.98)$ & $0.97^{\dagger}(0.95-0.99)$ \\
\hline Living situation & $<.01$ & $0.18^{\dagger}(0.08-0.44)$ & $0.73(0.43-1.22)$ & $0.25^{\dagger}(0.11-0.56)$ \\
\hline
\end{tabular}

ORs are presented with $95 \% \mathrm{Cl}$.

* Of care recipient.

${ }^{\dagger} P<.01$.

${ }^{\ddagger} P<.05$.

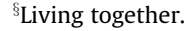

0.92-0.96) and living together (OR 0.18; CI 0.08-0.44) decreased this probability. The likelihood of being in the informal care class compared with the low user class is decreased by a higher score on the DAD (OR 0.97; 0.95-0.98) and increased by having a diagnosis of AD vs MCI (OR 1.84; CI 1.13-2.99) or dementia because of other causes vs MCI (OR 2.16; CI 1.22-3.83).

Although the entropy score was high, we additionally performed multinomial logistic regression weighted by the individuals' maximum class membership probability to account for uncertainty in class allocation. Findings showed no deviating results between the most likely class method and the weighted method.

\section{Discussion}

This study identified 3 classes of service use in a memory clinic population with a diagnosis of $\mathrm{MCI}$ and dementia. Classes mainly differentiated between the service types formal homecare (domestic and personal homecare), and informal care (ADL and IADL) and were, therefore, labeled as the "formal homecare class," "informal care class," and the "low user class." The largest class was the informal care class ( $46 \%$ of participants), and the smallest class the formal homecare class ( $10 \%$ of participants). Care recipient and informal caregiver not living together, older age of the care recipient, and a more impaired score on the DAD scale were strongly related to the formal homecare class. A diagnosis of dementia and impaired DAD score were strongly related to the informal care class.

We did not find any significant results in the univariate analyses for informal caregivers age, gender, and years of education on service use combinations, which is comparable to findings of Robinson et al. ${ }^{7}$ Gender of the care recipient was also not significant in the univariate analyses, although other studies found that gender is related to service use. ${ }^{6,40}$ We excluded NPI scores as a predictor in the main analyses. Other research indicated that behavioral changes are an important factor associated with social, healthcare, and informal care costs. ${ }^{4}$ However, NPI scores showed no significant result $(P=.154)$. One explanation for this might be a lack of variability because of the low NPI scores in our sample mainly in the subgroup of people with mild dementia. However, Herrmann et $\mathrm{al}^{41}$ found that even in a mildly impaired community-dwelling population NPI showed to be an important cost driver.

Living situation was significantly associated with being in the formal homecare class (ie, they were less likely to live together with their informal caregiver compared with the informal care class and low user class). This is not surprising giving the fact that caregivers who do live together with the care recipient often substitute activities that normally would be done by the formal care system. ${ }^{14,42}$

Furthermore, a higher care recipient's age was related to a higher likelihood of using formal homecare services, which is comparable to the findings of Beeber et al. ${ }^{19}$

Bergvall et $\mathrm{al}^{12}$ showed in their study that ADL measured by the DAD rather than cognition measured by the MMSE are the main predictor for informal care, which is comparable to our findings.

Lastly, as expected, care recipients with a diagnosis of dementia were more likely to use informal care compared with care recipients with $\mathrm{MCI}$. However, diagnosis was not related to using formal homecare in addition to informal care. This can be explained by the fact that other factors such as high care recipient age, impaired ADL functioning, and whether the care recipient and caregiver lived together showed a strong relation with service use and reflect the heterogeneity of care needs within the diagnostic syndrome categories of $\mathrm{MCI}$ and dementia.

This study has some limitations. First, this study was based on cross-sectional data and, therefore, determining causality or the ability to predict service use is limited. Second, service use was measured using a self-report questionnaire, which is less reliable than data retrieved by a face-to-face interview, ${ }^{19}$ and caregivers may have been unaware about services that were used. ${ }^{43} \mathrm{~A}$ third limitation is the use of a limited number of predictors. Including a broader range of demographic (eg, availability of nursing facilities), social (eg, attitude toward service use), and clinical predictors (eg, presence of comorbidities) is recommended for future studies as these have been shown to be related with service use.

\section{Implications for Practice and Research}

Our results showed that next to the syndrome diagnosis of $\mathrm{MCI}$ or dementia, age of the care recipient, ADL impairment, and living situation were also related to service use combinations. These findings confirm previous research and emphasize the importance to take these factors into account during the diagnostic process as these may improve to care planning. Future research should examine combinations of service use longitudinally, which makes it possible to actively prepare and plan the future need for services. A detailed understanding of the probability and interaction of care services over time could provide care professionals with the necessary information to form an individualized care plan and prepare persons with memory complaints for their future care need. Such information could also be of value for the coordination of care services to ensure uncomplicated transitions between care providers. 


\section{Conclusions}

This study showed that ADL impairment, not living together with the caregiver, and a higher age of the care recipient increased the likelihood of receiving formal homecare in memory clinic visitors with $\mathrm{MCI}$ and dementia. ADL impairment and having a dementia diagnosis increased the likelihood of receiving informal care. Therefore, these factors are relevant to take into account in addition to the diagnosis for care planning.

\section{Acknowledgments}

The authors thank everyone who contributed to, and participated in the LEARN study, 4C (dementia and MCI) study and the Dutch Flutemetamol study. Furthermore, the authors thank Nico Rozendaal for the technical support to perform this study and for the data management of the LEARN and $4 C$ studies. The LEARN study is performed within the framework of the Center for Translational Molecular Medicine, project LEARN (grant $02 \mathrm{~N}-101$ ). The 4C MCI study is funded by Alzheimer Nederland (grant 20083494), and the 4C Dementia study is funded by Alzheimer Nederland-VSB- foundation (grant 20083495).

\section{References}

1. Prince M, Bryce R, Ferri C. World Alzheimer Report 2011: The benefits of early diagnosis and intervention. London: Alzheimer's Disease International; 2011.

2. Wimo A, Jonsson L, Bond J, et al. The worldwide economic impact of dementia 2010. Alzheimers Dement 2013;9:1-11.e3.

3. Handels RL, Xu W, Rizzuto D, et al. Natural progression model of cognition and physical functioning among people with mild cognitive impairment and Alzheimer's disease. J Alzheimers Dis 2013;37:357-365.

4. Jonsson L, Eriksdotter Jonhagen M, Kilander L, et al. Determinants of costs of care for patients with Alzheimer's disease. Int J Geriatr Psychiatry 2006;21:449-459.

5. Luengo-Fernandez R, Leal J, Gray A. Dementia 2010: The prevalence, economic cost and research funding compared with other major diseases. A report prepared by the Health Economics Research Center, Oxford University, for the Alzheimer's Research Trust. Oxford: Alzheimer's Research Trust; 2010.

6. Boersma F, Eefsting JA, van den Brink W, van Tilburg W. Care services for dementia patients: Predictors for service utilization. Int J Geriatr Psychiatry 1997; 12:1119-1126.

7. Robinson KM, Buckwalter KC, Reed D. Predictors of use of services among dementia caregivers. West J Nurs Res 2005;27:26-140. discussion 141-147.

8. Scalmana S, Di Napoli A, Franco F, et al. Use of health and social care services in a cohort of Italian dementia patients. Funct Neurol 2013;28:265-273.

9. Chung SD, Liu SP, Sheu JJ, et al. Increased healthcare service utilizations for patients with dementia: A population-based study. PLoS One 2014:9:e105789.

10. Chen L, Reed C, Happich M, et al. Health care resource utilisation in primary care prior to and after a diagnosis of Alzheimer's disease: A retrospective, matched case-control study in the United Kingdom. BMC Geriatr 2014;14:76.

11. Weber SR, Pirraglia PA, Kunik ME. Use of services by community-dwelling patients with dementia: A systematic review. Am J Alzheimers Dis Other Demen 2011;26:195-204.

12. Bergvall N, Brinck P, Eek D, et al. Relative importance of patient disease indicators on informal care and caregiver burden in Alzheimer's disease. Int Psychogeriatr 2011;23:73-85.

13. Toseland RW, McCallion P, Gerber T, Banks S. Predictors of health and human services use by persons with dementia and their family caregivers. Soc Sci Med 2002;55:1255-1266.

14. Roelands M, Van Oost P, Depoorter A. Service use in family caregivers of persons with dementia in Belgium: Psychological and social factors. Health Soc Care Community 2008;16:42-53.

15. Lim J, Goh J, Chionh HL, Yap P. Why do patients and their families not use services for dementia? Perspectives from a developed Asian country. Int Psychogeriatr 2012;24:1571-1580.
16. Brodaty $\mathrm{H}$, Thomson $\mathrm{C}$, Thompson $\mathrm{C}$, Fine M. Why caregivers of people with dementia and memory loss don’t use services. Int J Geriatr Psychiatry 2005;20: 537-546.

17. Wolfs CA, de Vugt ME, Verkaaik M, et al. Empowered or overpowered? Service use, needs, wants and demands in elderly patients with cognitive impairments. Int J Geriatr Psychiatry 2010;25:1006-1012.

18. Rudin DJ. Caregiver attitudes regarding utilization and usefulness of respite services for people with Alzheimer's disease. J Gerontol Soc Work 1995;23: 85-108.

19. Beeber AS, Thorpe JM, Clipp EC. Community-based service use by elders with dementia and their caregivers: A latent class analysis. Nurs Res 2008;57: $312-321$.

20. Lopez OL. Mild cognitive impairment. Continuum (Minneap Minn) 2013;19: 411-424.

21. Petersen RC. Mild cognitive impairment as a diagnostic entity. J Intern Med 2004;256:183-194.

22. Zhu CW, Sano M, Ferris SH, et al. Health-related resource use and costs in elderly adults with and without mild cognitive impairment. J Am Geriatr Soc 2013;61:396-402.

23. Handels RL, Aalten P, Wolfs CA, et al. Diagnostic and economic evaluation of new biomarkers for Alzheimer's disease: The research protocol of a prospective cohort study. BMC Neurol 2012;12:72.

24. Oosterveld SM, Kessels RP, Hamel R, et al. The influence of co-morbidity and frailty on the clinical manifestation of patients with Alzheimer's disease. J Alzheimers Dis 2014;42:501-509.

25. Zwan MD, Bouwman F, Konijnenburg E, et al. Diagnostic impact of [18F]flutemetamol amyloid imaging in young-onset dementia. Alzheimer Dementia 2015;11:P3-P4.

26. Folstein MF, Folstein SE, McHugh PR. "Mini-mental state." A practical method for grading the cognitive state of patients for the clinician. J Psychiatr Res 1975; $12: 189-198$

27. Cummings JL. The neuropsychiatric inventory: Assessing psychopathology in dementia patients. Neurology 1997;48:S10-S16.

28. Gelinas I, Gauthier L, McIntyre M, Gauthier S. Development of a functional measure for persons with Alzheimer's disease: The disability assessment for dementia. Am J Occup Ther 1999;53:471-481.

29. Wimo A, Winblad B. Resource utilization in dementia: RUD Lite. Brain Aging 2003;3:48-59.

30. Asparouhov T, Muthén B. Using Mplus TECH11 and TECH14 to test the number of latent classes. Mplus Web Notes No.14; 2012. Available at: http:// www.statmodel.com/examples/webnotes/webnote14.pdf. Accessed March 30,2016

31. Schwarz G. Estimating the dimension of a model. Ann Stat 1978;6:461-464.

32. Lo Y, Mendell NR, Rubin DB. Testing the number of components in a normal mixture. Biometrika 2001;88:767-778.

33. Geiser C. Data analysis with Mplus. New York, NY: Guilford Publications; 2012.

34. McLachlan G, Peel D. Finite mixture models: Wiley series in probability and mathematical statistics. New York, NY: John Wiley \& Sons, Inc; 2000.

35. Nylund KL, Asparouhov T, Muthén BO. Deciding on the number of classes in latent class analysis and growth mixture modeling: A Monte Carlo simulation study. Structural Equation Modeling 2007;14:535-569.

36. Muthen LK, Muthen BO. Mplus user's guide. 6th. Los Angeles, CA: Muthén \& Muthén; 2010.

37. Ryan CJ, DeVon HA, Horne R, et al. Symptom clusters in acute myocardial infarction: A secondary data analysis. Nurs Res 2007;56:72-81.

38. IBM SPSS. Statistics for Macintosh. IBM Corp. Released 2013. Armonk, NY: IBM Corporation; 2013.

39. Clark SL, Muthén B. Relating latent class analysis results to variables not included in the analysis. 2009. Available at: https://www.statmodel.com/ download/relatinglca.pdf. Accessed March 29, 2016.

40. Kempen GI, Suurmeijer TP. Factors influencing professional home care utilization among the elderly. Soc Sci Med 1991;32:77-81.

41. Herrmann N, Lanctot KL, Sambrook R, et al. The contribution of neuropsychiatric symptoms to the cost of dementia care. Int J Geriatr Psychiatry 2006;21: 972-976.

42. Arno PS, Levine C, Memmott MM. The economic value of informal caregiving. Health Aff (Millwood) 1999;18:182-188.

43. Martindale-Adams J, Nichols LO, Zuber J, et al. Dementia caregivers' use of services for themselves. Gerontologist; 2015 Sep 8. pii: gnv121. [Epub ahead of print]. 